\title{
1 Genetic control of rhizosheath formation in pearl millet
}

2 De la Fuente Cantó C. ${ }^{1}$, Diouf M.N. ${ }^{2,3,7}$, Ndour P.M.S. ${ }^{2,3}$, Debieu M. ${ }^{1}$, Grondin A. ${ }^{1,4,5}$, Passot

3 S. ${ }^{1}$, Champion A. ${ }^{1}$, Barrachina C. ${ }^{6}$, Pratlong M. ${ }^{6}$, Gantet P. ${ }^{1}$, Assigbetsé K. ${ }^{2,3}$, Kane N. ${ }^{4}$, Cubry

4 P. ${ }^{1}$, Diedhiou A.G. ${ }^{4,7}$, Heulin T. ${ }^{8}$, Achouak W. ${ }^{8}$, Vigouroux Y. ${ }^{1}$, Cournac L. ${ }^{2}$, Laplaze L. ${ }^{1,4, *}$

$5{ }^{1}$ DIADE, Université de Montpellier, IRD, CIRAD, Montpellier, France

$6 \quad{ }^{2}$ Eco\&Sols, Université de Montpellier, IRD, CIRAD, INRAE, Institut Agro, Montpellier,

7 France

$8{ }^{3}$ Laboratoire Mixte International Intensification Écologique des Sols Cultivés en Afrique de

9 l'Ouest (IESOL), Dakar, Senegal

$10{ }^{4}$ Laboratoire Mixte International Adaptation des Plantes et microorganismes associés aux

11 Stress Environnementaux (LAPSE), Centre de recherche de Bel-Air, Dakar, Senegal

$12{ }^{5}$ CERAAS, Thiès, Senegal

$13{ }^{6}$ Montpellier GenomiX, Montpellier, France

$14{ }^{7}$ Département de Biologie Végétale, Faculté des Sciences et Techniques, Université Cheikh 15 Anta Diop, Dakar, Sénégal

$16{ }^{8}$ Aix Marseille Univ, CEA, CNRS, BIAM, LEMiRE, Laboratory of Microbial Ecology of the

17 Rhizosphere, ECCOREV FR 3098, Saint Paul-Lez-Durance, France F-13108

$18 *$ Author for correspondence: laurent.laplaze@ird.fr

19 Running title

20 Rhizosheath formation in pearl millet

22 Highlight

23 Formation of the rhizosheath, a layer of soil adhering to the root, is under complex genetic

24 control in pearl millet and is mainly regulated by root exudation. 


\section{Abstract}

26 The rhizosheath, the layer of soil that adheres strongly to roots, influences water and nutrients acquisition. Pearl millet is a cereal crop that plays a major role for food security in arid regions of sub Saharan Africa and India. We previously showed that root-adhering soil mass is a heritable trait in pearl millet and that it correlates with changes in rhizosphere microbiota structure and functions. Here, we studied the correlation between root-adhering soil mass and root hair development, root architecture, and symbiosis with arbuscular mycorrhizal fungi and we analysed the genetic control of this trait using genome wide association (GWAS) combined with bulk segregant analysis and gene expression studies. Root-adhering soil mass was weakly correlated only to root hairs traits in pearl millet. Twelve QTLs for rhizosheath formation were identified by GWAS. Bulk segregant analysis on a biparental population validated five of these QTLs. Combining genetics with a comparison of global gene expression in the root tip of contrasted inbred lines revealed candidate genes that might control rhizosheath formation in pearl millet. Our study indicates that rhizosheath formation is under complex genetic control in pearl millet and suggests that it is mainly regulated by root exudation.

\section{$41 \quad$ Keywords}

42 Arbuscular mycorrhizal fungi, Bulk segregant analysis, GWAS, Malate, Rhizosphere, Root 43 exudates, Root hairs, Soil aggregation

\section{Abbreviations}

RAS: root-adhering soil 


\section{Introduction}

Pearl millet is a small-seeded tropical cereal that was domesticated about 4,500 years ago in the Sahelian part of West Africa (Burgarella et al., 2018). It is mostly grown in dry and poor soils as a rainfed crop and is therefore well adapted to environments prone to drought and heat stress for which it harbours largely untapped genetic diversity in the locally adapted cultivated and wild pearl millets (Debieu et al., 2017; Varshney et al., 2017; Burgarella et al., 2018). The outstanding capacity for growing in harsh environments highlights the great potential of pearl millet as a biological model to investigate crop adaptation and resilience to abiotic constraints, as well as its key role for food security in some semi-arid tropical regions in Africa and Asia. Still, pearl millet yield remains low for two main reasons: the difficulty to reach its potential yield in constrained environments and the little attention that the crop has received from breeding programmes (Varshney et al., 2017).

Root traits are emerging as new targets for breeding more sustainable and resilient crop varieties in global climate change scenarios (Lynch, 2019). The root system is responsible for plant water and nutrient acquisition. Phenotypic selection of root ideotypes combining architectural, anatomical and physiological traits has been proposed as a way to optimise access to soil resources in specific agroecosystems and crop management practices (Lynch, 2019). Besides root architecture, anatomy and physiology, the rhizosphere, the volume of soil around the root influenced by the root (York et al., 2016), can be regarded as a plant extended phenotype and therefore a target for breeding more sustainable crops (Wissuwa et al., 2009; De la Fuente Cantó et al., 2020). Indeed, the dynamic interplay between root, soil and microbiota in the rhizosphere eases adaptation to changing environments and can have a remarkable impact on plant fitness (Turner et al., 2013; De la Fuente Cantó et al., 2020; Chai and Schachtman, 2021). The intricate relationships in the rhizosphere define a belowground niche where soil moisture, organic matter content, the composition of the microbial community and its activity are different from the bulk soil (Haichar et al., 2008; Hinsinger et al., 2009). Plants benefit from this interaction especially in constrained environments where access to nutrients and water is restricted (Yang et al., 2009).

The rhizosheath size, or root-adhering soil mass, is a proxy in the study of this complex extended phenotype and an interesting potential target for breeding programmes (Ndour et al., 2020). Rhizosheath formation was first noticed as the sandy sheath surrounding the roots of desert plants (Price, 1911) and later reported to occur across many angiosperm orders (Brown 
et al., 2017). Increased rhizosheath size has been correlated with enhanced wheat and foxtail millet performance in drying soils (Basirat et al., 2019; Liu et al., 2019). In barley and oat, rhizosheath formation has been related with improved acquisition of major and essential trace elements in limiting water conditions (Nambiar, 1976; George et al., 2014). A combination of root architectural and anatomical traits and the secretion of root exudates and mucilage have been connected to soil aggregation to the root (Pang et al., 2017; Ndour et al., 2020). For instance, root branching, root hair formation or symbiosis with arbuscular mycorrhizal fungi (AMF) have been associated to some extent with rhizosheath establishment (MorenoEspíndola et al., 2007; Brown et al., 2017; Liu et al., 2019). Root architectural traits have been found crucial for rhizosheath formation in wheat and foxtail millet (Delhaize et al., 2012; Liu et al., 2019). On the other hand, root exudates composition and mucilaginous polymers released by root-associated microorganisms impact the stability of soil aggregates that bind around the root (Galloway et al., 2020). Root growth and exudates exert dynamic changes in the rhizosphere physical properties and hydraulic processes that affect soil nutrient dynamics and the composition of the rhizosphere associated microbiota (Dakora and Phillips, 2002; Kolb et al., 2017; Sasse et al., 2018; Chai and Schachtman, 2021). Despite the inherent complexity linked to the effect of exudates in the rhizosphere, some studies showed their direct relationship with rhizosheath formation. For example, greater mass of mucilage exuded by chickpea roots were linked with the formation of larger and more porous rhizosheaths capable of storing more soil moisture in drought tolerant cultivars (Rabbi et al., 2018). In annual crops such as wheat, barley and maize, there is evidence of the remarkable plant genetic influence in the formation of rhizosheath and the processes of rhizodeposition influencing rhizosphere microbial activities (George et al., 2014; Delhaize et al., 2015; Mwafulirwa et al., 2016; 2021b), however few studies have aimed to dissect the genetics underlying the conformation of this extended root phenotype (George et al., 2014; Delhaize et al., 2015; James et al., 2016; Mwafulirwa et al., 2021a).

In previous studies, we reported a remarkable genotypic variability for root-adhering soil aggregation in pearl millet (Ndour et al., 2021). Moreover, this variability was associated with changes in rhizosphere microbiota structure and function (Ndour et al., 2017, 2021). Here, we analysed the relative contribution of root architectural characteristics and root colonization by AMF on root-adhering soil aggregation in pearl millet. We then combined a genome wide association analysis (GWAS), with bulk segregant analysis (BSA) and transcriptomic data to dissect the genetic bases of this complex trait. 


\section{Materials and methods}

\section{Plant materials}

115 A panel of 181 pearl millet inbred lines developed at the International Crops Research Institute

116 for the Semi-Arid Tropics (ICRISAT, Niger) from landraces and improved open-pollinated

117 cultivars representing the genetic diversity of the crop in West and Central Africa was used in

118 this study (Debieu et al., 2018).

119 Two inbred lines from this panel with contrasted rhizosheath size measured by the ratio 120 between the mass of root-adhering soil (RAS) and root biomass (RT; RAS/RT ratio; Ndour et 121 al., 2021): ICML-IS 11139 (small rhizosheath size parent) and ICML-IS 11084 (large 122 rhizosheath size parent) were selected for a bi-parental cross. The obtained F2 offspring was 123 then used in a bulk segregant analysis (BSA).

\section{Plant growth and measurement of soil aggregation}

125 Plants were grown for 28 days in "WM" shaped pots (WM 20-8-5, Thermoflan, Molières126 Cavaillac) containing $1.5 \mathrm{~kg}$ of soil under natural light in a greenhouse in the ISRA/IRD Bel 127 Air Campus in Dakar (Lat. 14.701778, Long. -17.426229, altitude $9 \mathrm{~m}$ ) as previously described 128 (Ndour et al., 2021).

129 For the GWAS analysis, pearl millet lines were sown according to a complete random block

130 design with seven repetitions. Thinning was performed to have one plant per pot. Soil moisture 131 was adjusted daily at water-holding capacity. Plant watering was stopped 24 hours before 132 harvesting to facilitate the separation of root-adhering soil (RAS) from bulk soil. Plants were 133 harvested 28 days after planting by opening the pots gently and shaking the plant and its 134 adhered soil at a constant speed (1100 rpm) for $1 \mathrm{~min}$ with a CAT S50 electric shaker (Cat 135 Ingenieurbuero ${ }^{\mathrm{TM}}$ ) to separate the bulk soil from the RAS uniformly. Roots were then rinsed 136 in a cup with demineralized water to collect RAS. The RAS was dried at $105^{\circ} \mathrm{C}$ for three days 137 and weighted. Roots and shoots were separated and dried at $65{ }^{\circ} \mathrm{C}$ for three days. The ratio 138 between mass of RAS and mass of root tissue (RT; RAS/RT) was used to estimate the 139 rhizosphere aggregation intensity (rhizosheath size) as previously described (Ndour et al., 140 2017). 
141 For BSA analysis, RAS/RT ratio was measured on 553 F2 individuals grown in five successive 142 blocks of 119, 112, 131, 130 and 61 F2 plants. Each of these blocks included six replicates 143 randomly distributed for each parental line. At the end of the experiment, leaf disk samples of $1441.5 \mathrm{~mm}$ diameter were sampled for each individual plant and stored at $-80{ }^{\circ} \mathrm{C}$ for genotyping.

145 For correlation analyses between RAS/RT ratio and related root traits (root architecture, root 146 hair length and density and interactions with AMF), 8 contrasting genotypes for rhizosheath 147 size were analysed in 2018 and 2020 ( $n=10$ plants/genotype in 2018 and $n=6$ plants/genotype 148 in 2020).

\section{Root architecture}

150 Root architecture traits (length, average diameter, total root area) were measured using the 151 WinRHIZO software (version 2012b) after scanning the roots using an Epson Perfection V700 scanner. Roots were separated in two groups based on their diameter according to Passot et al.

153 (2016): primary and crown roots $(0.25 \mathrm{~mm}<$ diameters $)$ and lateral roots (diameters $<0.25$ $154 \mathrm{~mm})$.

155 Root hair length and density were measured on four plants per genotype using images of the 156 root hair zone of three lateral roots per plant. Images were taken using an optical microscope 157 (BX50F, Olympus) equipped with a digital camera (Micro Publisher 3.3 RTV). For each lateral 158 root, the total number of root hairs was recorded over a distance of $0.5 \mathrm{~mm}$ using the Mesurim 159 free software (http://acces.ens-lyon.fr/acces/logiciels/applications/mesurim/mesurim) and the 160 length of 10 randomly selected root hairs was measured using the ImageJ software.

\section{Root colonization by arbuscular mycorrhizal fungi}

162 Intensity and frequency of root colonization by AMF were measured according to Trouvelot et al., (1986) after roots staining with Trypan blue following the method described by Phillips and Hayman (1970). Stained root fragments were observed with a Nikon Labophot trinocular microscope. For each fragment, a score between zero and five was assigned according to the estimated proportion of root cortex colonized by AMF (Trouvelot et al., 1986).

167 Frequency and intensity of root colonization were then computed using the following formulas: 
where $\mathrm{n}$ is the number of fragments showing mycorrhizae and $\mathrm{N}$, the number of observed fragments the number of fragments observed.

\section{Heritability}

$$
H^{2}=\frac{\operatorname{Var}(\text { line })}{\operatorname{Var}(\text { line })+\frac{\operatorname{Var}(\text { res })}{n_{\text {plant/line }}}},
$$

where $n_{\text {plant/line }}$ is the average number of plants measured per line, $\operatorname{Var}($ line $)$ is the variance associated with lines and $\operatorname{Var}($ res $)$ is the residual variance.

Both variances are parameters of the following linear mixed model:

$$
\mathrm{RSA}=\mu+\alpha_{\text {line }}+\varepsilon_{\text {res }}
$$

181 where $\mu$ is the overall mean soil aggregation, $\alpha_{\text {line }}$ is the random effect attached to the lines with

$$
\alpha_{\text {line }} \sim \mathrm{N}(0, \operatorname{Var}(\text { line })) \text { and } \varepsilon_{\text {res }} \text { is the error term with } \varepsilon_{\text {res }} \sim \mathrm{N}(0, \operatorname{Var}(\text { res })) \text {. }
$$

\section{Genome wide association mapping and statistical analysis}

184 Genotyping by sequencing of this panel of inbred lines was previously reported (Debieu et al., 2018). As a preliminary step, we used the genotypic matrix to estimate the population structure.

186 Individual ancestry coefficients were estimated using the R package LEA v2.0 (Frichot and

187 François, 2015). We used a latent factor mixed model (LFMM) that considers ridge estimates 188 and corrects for unobserved population cofounders, i.e. latent factors, to perform the GWAS 189 (Caye et al., 2019). In addition, we ran the efficient mixed-model association (EMMA, Kang 190 et al., 2008) and mixed linear model (MLM) implemented in the R package GAPIT (Lipka et $a l ., 2012)$ to contrast the results. The proportion of the phenotypic variance explained by a QTL was determined by estimating the $\mathrm{R}^{2}$ corrected for population structure of a linear model defined for the most significant SNPs. 


\section{Bulk Segregant Analysis}

NGS-based BSA studies require establishing contrasted groups or bulks of lines to assess the differences in segregation of alleles using high-throughput sequencing (K. L. Nguyen et al., 2019). The $10 \%$ extreme lines in the tails of the phenotype distribution for the RAS/RT ratio were selected and the corresponding leaf discs were pooled to form bulks of contrasted lines. Genomic DNA was isolated for each bulk using a MATAB (Mixed Alkyl Trimethyl Amonium Bromide) based method (Mariac et al., 2006) and enriched DNA libraries were constructed for which 32,860 predicted genes from the pearl millet reference genome (Varshney et al., 2017) were targeted using gene capture probes (myBaits ${ }^{\circledR}$ ). High-throughput sequencing of the enriched DNA library was performed on an Illumina HiSeq platform by Novogene Company Limited (HK). Initial sequencing quality checks using FastQC version 0.11.5 (Andrews, 2010) were followed by trimming and quality filter steps on which adaptors, barcode sequences and low-quality reads $(<35$ bp) were removed. Paired sequences were then retained and aligned to the pearl millet reference genome using the BWA MEM algorithm (BWA version 0.7.17 - r1188, Li and Durbin, 2009). Reads mapping at the target-enriched regions were used for SNP calling using the UnifiedGenotyper algorithm from GATK 3.7 (McKenna et al., 2010). Down-sampling limit (dcov) was increased from the default value of 250 to 9000 to ensure accounting for the maximum coverage reached at each position. Multi-

212 allelic sites and those which exhibited a total allele frequency less than 0.25 were removed. In 213 addition, sites with either low or high total sequencing depth (below the $25^{\text {th }}$ and above the $95^{\text {th }}$ 214 percentiles respectively) were removed. SNPs with more than 50\% missing data and minor 215 allele frequency (MAF) under 5\% were also excluded. Finally, the parental line ICML-IS 21611139 (low RAS/RT ratio) was used as the reference genome for the cross to designate the alternate and reference SNP variants in the bulks.

218 Euclidean distance-based statistics (Hill et al., 2013) was used to measure the difference in 219 allele frequency between the bulks. The Euclidean distance between allele frequencies of the bulks at each marker position $(E D m)$ was calculated as follows:

$$
E D_{m}=\sqrt{\left(f_{a L}-f_{A L}\right)^{2}+\left(f_{a H}-f_{A H}\right)^{2}}
$$
low bulk (L) and the high bulk $(\mathrm{H})$ respectively. 
In order to reduce the effect of sequencing noise and increase the signal of the differences in allele frequency, we then calculated the fourth power of the cumulative EDm value in windows of 100 consecutive markers (Omboki et al., 2018; Zhang et al., 2019). The confidence interval of the statistic was determined using simulations as described (de la Fuente Cantó and Vigouroux 2021, under revision)

\section{Gene expression analyses}

Seeds from lines ICML-IS 11139 (low RAS/RT ratio) and ICML-IS 11155 (high RAS/RT ratio) were surface-sterilized and germinated in Petri dishes containing wet filter paper for 24 $\mathrm{h}$ in the dark at $27^{\circ} \mathrm{C}$. After two days, plants were transferred to hydroponic tanks containing

234 liquid half Hoagland solution and grown for 15 days at $27^{\circ} \mathrm{C}(12 \mathrm{~h}$ light/12 h dark). RNA was extracted from crown root tips (two cm apex) using the RNeasy Plant Mini Kit (QIAGEN).

236 RNA-seq was performed by the Montpellier GenomiX Platform (MGX, 237 https://www.mgx.cnrs.fr/). Sequencing was performed on an Illumina HiSeq 2500. Three different statistical tests were used to identify differentially expressed genes: EdgeR (Robinson et al., 2010), DESeq (Anders and Huber, 2010) and DESeq2 (Love et al., 2014). GO terms enrichment was performed in the 1270 genes that were significantly differentially expressed between the two lines for all three statistical tests using the TopGO package in R.

\section{Statistical methods}

243 All statistical analyses were performed with $\mathrm{R}$ version 3.6.3 (R core Team, 2018).

\section{Results}

\section{Root-adhering soil aggregation is weakly correlated to root hair traits in pearl millet}

Several root traits have been proposed to contribute to root-adhering soil aggregation (as an integrative phenotype) including root hair development, root architecture, and arbuscular mycorrhizal symbiosis. We therefore analysed the contribution of these different traits to rootadhering soil aggregation in pearl millet. For this, we analysed correlation between root- 
intensity of root colonization by AMF in eight inbred lines with contrasted rhizosphere aggregation phenotype (Ndour et al., 2021) after four weeks of growth. Among root architecture traits, only the average root diameter (AvgDiam) was weakly and negatively correlated $\left(p=0.012, r^{2}=0.057\right)$ with root-adhering soil aggregation (Fig. 1A, Table 1). For root hairs, only average length of root hairs (AvgLRH) was weakly and positively correlated to root-adhering soil aggregation $\left(p=0.005, r^{2}=0.077\right.$; Fig. 1A, Table 1$)$. No significant correlation was observed for all other traits including frequency and intensity of root colonization by AMF (Fig. 1A, Table 1). Similar results were found in two independent experiments (2018 and 2020; Supplementary Table S1 at JXB online).

Altogether, our results suggest that root hairs development could play a weak role in rootadhering soil aggregation in pearl millet and that root architectural traits and AMF colonization rate have no significant impact.

\section{Genetic bases of rhizosheath formation in pearl millet}

We previously reported the phenotyping of a panel of pearl millet inbred lines for rootadhering soil aggregation (Ndour et al., 2021). Briefly, a total of 1408 plants corresponding to 181 inbred lines were phenotyped and we recorded an almost four-fold variation in rhizosheath size (RAS/RT ratio), ranging from 7.4 (ICML-IS 11139) to 26.3 (ICML-IS 11084; Ndour et al., 2021). Here, we used these data to evaluate the heritability of root-adhering soil aggregation. A broad sense heritability of 0.72 was computed, suggesting that root-adhering soil aggregation is largely under genetic control. Altogether, these data indicate that rootadhering soil formation has high heritability and that a large genetic diversity exists in pearl millet.

We therefore analysed the genetic bases of root-adhering soil formation using association genetics. Out of the 181 inbred lines, 139 lines with good quality data for phenotype and genotype were retained to perform the association study. As a first step, we conducted a population structure analysis of the 139 lines (Supplementary Fig. S1) that confirmed the negligible genetic structure previously reported for this panel (Debieu et al., 2018). A total of 381,899 SNPs was used for the association analysis. We first calculated the least square means of the trait root-adhering soil aggregation (RAS/RT ratio) across the different experiments. The ratio ranged from 12.4 to 26.3 with an average of 18.0. The LFMM model for GWAS identified 53 significant SNPs ( $p$-value $<0.0001)$ across the genome (Fig. 2A), defining 34 significant 
regions or QTLs considering windows of $50 \mathrm{~kb}$ up and downstream of significant positions to define significant regions. The proportion of phenotypic variance accounted for the most significant SNPs defining QTLs ranged from 9.2 to $15.6 \%$ indicating that the corresponding QTLs had small phenotypic effect.

We compared these results with two other GWAS methods (Fig. 2B\&C). Thirty-nine of these SNPs included in 25 QTLs defined through LFMM were also found significant with EMMA, and 19 significant SNPs assigned to 14 QTLs using MLM model in GAPIT (Supplementary Table S2). Fifteen SNPs in 12 QTL regions were found significant across the three GWAS methods. Altogether, our GWAS analysis revealed 12 potential QTLs controlling root-adhering soil aggregation in pearl millet.

To back up our GWAS analysis, we performed bulk segregant analysis in a F2 population derived from a cross between two lines with contrasted RAS/RT phenotypes, ICML-IS 11139 (low RAS/RT) and ICML-IS 11084 (high RAS/RT; Fig 3A). F2 plants were phenotyped in five consecutive blocks together with the parental inbred lines. We confirmed the contrasted RAS/RT ratio of the parental lines with average values of 15.0 (ICML-IS 11139) and 32.7 (ICML-IS 11084, Fig. 3B). Ten individual F2 lines were dropped from the analysis leaving $547 \mathrm{~F} 2$ with RAS/RT ratio ranging from 1.6 to 54.8 and with an average value of 22.3. The phenotype distribution of the F2 was slightly skewed towards high values of RAS/RT ratio and showed a significant block effect (Supplementary Fig. S2). We used log transformation of RAS/RT ratio in our analysis of variance and selected lines with extreme values of the residual term for the bulks. The bulks consisted in two groups of $55 \mathrm{~F} 2$ lines each, with RAS/RT ratio average values of 11.0 for the low RAS/RT bulk and 38.2 for the high RAS/RT bulk (Supplementary Table S3). A total of 223.6 Mbp reads were mapped to the target enriched regions and used for SNP calling. We identified a group of 23,160 SNP variants (1.5 SNPs per $100 \mathrm{~kb}$ in average) between the bulks. The average sequencing depth was high with $887 \mathrm{X}$ and $863 \mathrm{X}$ in the small and high RAS/RT bulk respectively. The NGS-based BSA analysis revealed significant differences in the allele frequency of 380 SNPs at the $95 \%$ confidence interval (Table 2, Fig 3C). These SNPs defined five significant chromosome regions linked to the segregation of the RAS/RT ratio phenotype: three on chromosome 5 (RAS5.1, RAS5.2 and RAS5.3) and two on chromosome 6 (RAS6.1, RAS6.2; Table 2). The smallest genomic region defined corresponded to RAS5.3 with $10.6 \mathrm{Mbp}$ and 41 significant SNPs. In contrast, the largest significant region corresponded to RAS5.2, with $45 \mathrm{Mbp}$ and 307 significant SNPs. 
Interestingly, the range of four out of five BSA significant regions was found to overlay with the position of significant SNPs defined by GWAS (Figure 3C, Supplementary Table S2).

317 Furthermore, the peak position of RAS5.1 on chromosome 5 is located $43 \mathrm{~kb}$ away from the 318 SNP chr5_3282686 identified by GWAS (LFMM and EMMA). Likewise, the RAS5.3 spans 319 through a genomic region containing two GWAS QTLs (LFMM, MLM and EMMA) located $113 \mathrm{~kb}$ and $244 \mathrm{~kb}$ away from the peak position of RAS5.3.

Altogether, the combination of GWAS and BSA analyses revealed genomic regions on chromosomes 5 and 6 controlling RAS/RT ratio in pearl millet.

\section{Comparison of gene expression in contrasted lines}

To further analyse the genes involved in rhizosheath formation, we compared gene expression in ICML-IS 11139 (low RAS/RT) and ICML-IS 11155 (high RAS/RT) roots. Production and secretion of root exudates occur along the root system (Haichar et al., 2014), starting in the zone immediately behind the root tip (Schroth and Snyder, 1962). Similarly, root hair development occurs in the root tip. Thus, as these two processes seem to be the major determinants of root-soil aggregation in pearl millet, we hypothesized that genes controlling this trait might be preferentially expressed in the root tip. Phenotyping for RAS/RT ratio was performed at 28 days after planting, when the root system of pearl millet was made of one primary root and several crown roots possessing lateral roots (Passot et al., 2016). As crown roots make up most of the root system at this stage and to avoid noise due to sample heterogeneity (different root types), we therefore compared gene expression in the crown root tips ( $2 \mathrm{~cm}$ apex) of the two contrasted lines. RNAseq revealed 1270 genes with significant differences in gene expression between the two contrasted lines using three combined statistical tests (EdgeR, DESeq et DESeq2, $p$-value < 0.05; Supplementary Fig. S3). A gene ontology analysis on 742 genes with GO annotation out of the 1270 differentially expressed genes revealed a significant enrichment in GO terms associated with proteins involved in molecular interactions (GO:0043531, ADP binding with lowest $p$-value) and enzymatic reactions (GO:0016706, oxidoreductase activity for instance; Supplementary Table S4).

\section{Candidate genes analysis}

343 We combined GWAS, BSA and gene expression analyses to identify candidate genes for RAS 344 aggregation. We first focused our search for candidate genes in the QTL regions identified by 345 GWAS that were coincident with regions of significance defined through BSA on 
chromosomes 5 and 6 (GWAS QTLs 5.1, 5.3, 5.5, 5.6 and 6.3). We assessed the annotated genes from the reference genome (Varshney et al., 2017) included in a $1 \mathrm{Mbp}$ region centred around the most significant SNP position together with their expression data from the RNAseq experiment.

The most significant SNP marker in GWAS QTL 5.1 maps in chromosome 5 position 3,282,686 bp in an intergenic region between a cluster of four genes coding for glyoxylate reductase (Pgl_GLEAN_10016760, Pgl_GLEAN_10016761,Pgl_GLEAN_10016762 and Pgl_GLEAN_10016764). Out of the four genes, one was differentially expressed in the contrasted lines for RAS/RT, the others showed a weak and variable expression level within the same genotype. Glyoxylate reductases are recycling enzymes that reduce glyoxylate to glycolate (Hoover et al., 2007). Interestingly, the glyoxylate cycle plays an important role in the synthesis of malate, which is a major metabolite excreted in root exudates (Fernie and Martinoia, 2009). This region also contains Pgl_GLEAN_10016765, a gene coding for an arginase with significantly higher expression in ICML-IS 11139 (low RAS/RT). Arginases metabolise arginine and provide nitrogen for the synthesis of other essential amino acids during plant development and stress response mechanisms (Siddappa and Marathe, 2020). Large variations in arginine concentrations have been associated with changes in root exudate composition in plants exposed to drought (Gargallo-Garriga et al., 2018).

The GWAS QTLs 5.5 and 5.6 are coincident with the same region of significance defined in BSA, RAS5.3. This 10.56 Mbp region contains 105 annotated genes in the reference genome. Interestingly, the most significant marker trait association for GWAS QTL 5.5 falls into a gene showing some homology to remorins (Pgl_GLEAN_10037821). Remorins are membrane proteins playing an important role in plant biotic interactions (Jarsch and Ott, 2011).

The most significant SNP in GWAS QTL 6.3 maps in chromosome 6 position 227,616,229 bp in a gene coding for a galactinol-sucrose galactosyltransferase that is more expressed in the low RAS/RT ratio line (Pgl_GLEAN_10028942). These are enzymes involved in the synthesis of raffinose (Lehle and Tanner, 1973), an oligosaccharide stored principally in seeds, roots and tubers. Accumulation of raffinose in wheat and tomato roots occurs in response to low $\mathrm{P}$ conditions (Sung et al., 2015; V.L. Nguyen et al., 2019). Raffinose also accumulates in roots of pea seedlings exposed to water stress (Lahuta et al., 2014). In addition, the secretion of this oligosaccharide in root exudates is linked to the complex biotic interactions in the rhizosphere (Fang and Leger, 2010; Liu et al., 2017). 
We also looked for candidate genes associated with the most significant SNPs consistently identified by GWAS that do not coincide with regions of significance defined through BSA. The GWAS QTL 2.3 contains a cluster of five significant SNPs mapping in the same gene, Pgl_GLEAN_10019483, encoding an LRR receptor-like serine/threonine-protein kinase. This gene is strongly expressed in the root tip of both pearl millet lines. LRR receptor kinases are involved in the perception of signalling molecules (Chakraborty et al., 2019).

The GWAS QTL 6.2 consists of two SNPs markers on chromosome 6 mapping in an intergenic region between a cluster of four genes coding for acidic endochitinase (Pgl_GLEAN_10020193, Pgl_GLEAN_10020194, Pgl_GLEAN_10020195 and Pgl_GLEAN_10020196), one of them with higher expression in the low aggregation line, the others with similar or weak expression in both lines. Endochitinase and chitinase-like proteins are defence related proteins with anti-fungal activity that are found in root exudates of different plant species (Nóbrega et al., 2005; Tesfaye et al., 2005; De-la-Peña et al., 2010).

In chromosome 7, we found a group of five significant SNP markers within a $74 \mathrm{~kb}$ range defining the GWAS QTL 7.5. These SNPs were close to a gene encoding a putative chloroplastic dicarboxylate transporter that exchanges malate for succinate, fumarate and 2oxoglutarate $\left(P g l \_G L E A N_{-}\right.$10006630). All these are important components of root exudates. The region also contains a gene coding for an $\mathrm{ABC}$ transporter $\mathrm{G}$ family member (Pgl_GLEAN_10006636). ABC transporters are involved in the transport of root exudates (Badri et al., 2009; Baetz and Martinoia, 2014).

\section{Discussion}

Here, we investigated root system architectural traits with potential impact on rhizosheath formation in pearl millet. The presence of root hairs is essential for rhizosheath formation but the impact of root hair length and density on rhizosheath size varies considerably between plant species (Brown et al., 2017). Studies in wheat and maize showed that root hair length is strongly correlated with rhizosheath weight (Delhaize et al., 2012; Adu et al., 2017). Similarly, in foxtail millet, increased rhizosheath formation was found related with the plastic response in root hair formation (increases in root hair elongation and density) in dry soils (Liu et al., 2019). This relationship was not as clear in crops such as barley (George et al., 2014). However, a recent study shows an increased rhizosheath formation in barley grown in drying soil associated with auxin-promoted growth of root and root hairs as a consequence of ABA 
accumulation (Zhang et al., 2021). In our study on pearl millet, we have identified a weak but significant correlation between rhizosheath formation, which is synonymous with rootadhering soil formation in this work, and root hair length $\left(p=0.005, r^{2}=0.077\right)$. This suggests that root hairs are involved in rhizosheath formation in pearl millet but that they play a limited role in our experimental conditions.

Root association with arbuscular mycorrhizal fungi (AMF) has been proposed to contribute to rhizosheath formation (Pang et al., 2017). In our study, we did not find any correlation between rhizosheath formation and AMF colonization rate in pearl millet suggesting that AMF colonization level is not an important driver in rhizosphere aggregation in this species.

Altogether, we hypothesise that rhizosheath formation or the aggregation of soil particles to the root in pearl millet is mainly driven by other traits. Root exudates and mucilaginous polymers released by root-associated microorganisms as well as the enzymatic activities linked to the crosstalk interactions occurring in the rhizosphere are prime candidates. Accordingly, the different orders of bacteria predominantly found in the rhizosphere of pearl millet lines with contrasted root soil aggregation suggests that the differences in rhizosheath formation could be linked to crosstalks between the plant and microbial community (Ndour et al., 2017, 2021). Further work will be needed to test this hypothesis.

In the current study, the large variation in rhizosheath size in a genetically diverse group of inbred lines revealed a high heritability value for the trait $\left(\mathrm{H}^{2}=0.72\right)$. Although rhizosheath formation relies on a range of traits mainly related with root morphology and exudates, it has been found under genetic control in other cereal crops such as wheat (Delhaize et. al., 2015; James et al., 2016) and barley (George et al., 2014; Gong and McDonald, 2017), becoming a potentially interesting target trait for breeding (Ndour et al., 2020). Chromosome regions associated with rhizosheath size were identified in both crops, however few candidate genes underlying the QTL regions have been proposed. Interestingly, comparative evaluation of the multiple loci identified in these studies shows a lack of QTLs identified across diverse growing conditions suggesting, to some extent, a large QTL by environment interaction likely linked to the plasticity of rhizosheath formation.

Here, the combination of GWAS and BSA allowed the identification of four chromosome regions controlling rhizosheath size in pearl millet and ultimately some putative candidate genes based on gene annotations in the reference genome (Varshney et al., 2017). GWAS 
440 allowed the identification of 34 significant QTLs using the latent factor mixed model or LFMM

441 (Caye et al., 2019) method. Many of these associations were confirmed using two other models

442 for GWAS analysis (EMMA and MLM). The phenotypic variance explained (PVE) by these

443 loci ranged from $11.2 \%$ to $14.7 \%$ suggesting that rhizosheath size as a complex trait determined

444 by many QTLs of moderated effect in pearl millet. Consistently, studies in biparental and

445 multiparental populations of wheat revealed several QTLs linked to rhizosheath formation with

446 proportions of variation explained by QTLs around 5 to 10\%. (Delhaize et. al., 2015; James et

447 al., 2016). Nonetheless, one major QTL for the trait was also identified in wheat (James et al.,

448 2016).

449 Few genetic studies have identified genes potentially involved in rhizosheath formation and

450 their predicted functions were mainly linked to root system morphogenesis and growth. For

451 example, root hair length is a major driver determining rhizosheath size in wheat and,

452 accordingly, genes coding for basic helix-loop-helix family of transcription factors that are

453 known to control root hair development were identified as potential candidates underlying a

454 rhizosheath QTL in that species (Delhaize et. al., 2015). In barley, genes controlling cell

455 division in root apical meristem at seedling stage and genes linked to tolerance to drought and

456 cold were also identified as putative candidates underlying some genomic regions associated

457 with rhizosheath size (George et al., 2014).

458 In contrast, in our study, candidate genes were mostly related to plant metabolism and transport.

459 Combining BSA and GWAS analyses revealed five co-localizing QTL regions. Candidate

460 genes in these QTLs regions were mostly linked with root metabolic activities such as the

461 synthesis of compounds commonly found in root exudates. For instance, the glyoxylate

462 reductase and the arginase identified as putative candidates for QTL 5.1 are involved in the

463 reduction and storage of essential compounds (i.e., glyoxylate and nitrogen) required for

464 metabolic processes that mediate the synthesis of organic acids like malate and the synthesis

465 of amino acids, respectively (Igamberdiev and Eprintsev, 2016; Siddappa and Marathe, 2020).

466 These are major primary metabolites of root exudates which variations in concentration can

467 trigger plant adjustments to enhance root access and mobilisation of soil phosphate and

468 nitrogen when these nutrients are limited (Carvalhais et al., 2011; Mora-Macías et al., 2017;

469 Canarini et al., 2019). Further, these compounds have been found to promote chemotaxis of

470 beneficial bacteria into the rhizosphere (Feng et al., 2018). In fact, a recent study showed how

471 differences in malate concentration in root exudates impacted the composition of microbial 
communities associated with wheat and rice root systems (Kawasaki et al., 2021). Another potential candidate gene identified for QTL 6.3, a galactinol-sucrose galactosyltransferase, is involved in the synthesis of raffinose, an oligosaccharide which variations in concentration in root exudates has been found to favour root colonisation by rhizosphere microbes (Liu et al., 2017).

477 Our genetic analysis is therefore fully consistent with our analysis showing that root

478 architectural, root hair and AM symbiosis traits are not or poorly correlated with rhizosheath

479 formation in pearl millet, and with our expression study that shows that genes involved in plant metabolism are differentially regulated between lines with contrasted rhizosheath size. It is also consistent with previous research showing differences in the rhizosphere metabolic activity of pearl millet lines with contrasted rhizosheath size (Ndour et al., 2021). In this study, increased activity of enzymes such as chitinase and phosphomonoesterase was observed in the rhizosphere of pearl millet lines with larger rhizosheath (same contrasted RAS/RT lines used in the present work). We hypothesised that increased exudation in lines with larger rhizosheath size lead not only to an enhanced stability of root-adhering soil aggregates but also to a decrease of $\mathrm{pH}$ that could have stimulated these enzyme activities (Ndour et al., 2021). Moreover, the amount of root exudate and the function of these enzymes could also impact the rhizosphere microbial communities promoting rhizosheath formation and explain the difference found in microbiota diversity in contrasted pearl millet lines (Ndour et al., 2021).

491 In conclusion, our physiological and genetic analysis suggest a central role for root exudation

492 (quantitatively or qualitatively) in the regulation of rhizosheath formation in pearl millet.

493 Rhizosheath formation seems to be controlled by many QTLs with small effects. We identified several candidate genes controlling this trait and future work will focus on the validation and characterization of the molecular mechanisms regulating rhizosheath formation in pearl millet.

\section{Supplementary data}

498 Figure S1. Ancestry estimation using the cross-entropy criterion.

499 Figure S2. Frequency distribution of the RAS/RT phenotype in the bi-parental population 500 designed for BSA experiment. 
Figure S3. Number of genes differentially expressed in two contrasted inbred lines.

Table S1. Spearman correlation between the different traits in 2018 and 2020 experiments.

503 Table S2. Significant marker-trait associations for root-adhering soil aggregation using 3

504 GWAS methods

Table S3. RAS/RT phenotype in the contrasted inbred lines and the bulks used for BSA.

Table S4. Top ten GO enriched terms in genes differentially expressed between contrasting inbred lines.

\section{Acknowledgements}

509 This work was supported by the French National Research Institute for Sustainable

510 Development (IRD), the French Agence Nationale de la Recherche (ANR grant RootAdapt 511 ANR17-CE20-0022-01 to LL), the CGIAR Research Programme on Grain Legumes and 512 Dryland Cereals (GLDC), the NewPearl grant in the frame of the CERES initiative by the 513 Agropolis Fondation (AF 1301-015 to LL as part of the 'Investissement d'avenir' ANR-10514 LABX-0001-01 under the frame of I-SITE MUSE ANR-16-IDEX-0006), and by the 515 Fondazione Cariplo (No FC 2013-0891).

\section{Authors contributions}

518 YV, LC and LL conceptualized and supervised the research; LL acquired the funding. CFC, 519 MND, PMSN, MD, AG, SP, CB, and MP carried out the measurements and performed formal analyses. All authors discussed and evaluated the data. CFC, MND, PMSN, AG, YV, LC and LL wrote the first draft of the manuscript; all authors revised the manuscript and gave final approval for publication.

\section{Data Availability}

525 The data that support the findings of this study are openly available at the National Center for 526 Biotechnology Information (NCBI). Genotyping (GBS) data are available in genbank under 527 reference number PRJNA492967 (GWAS) and PRJNA769524 (BSA). RNAseq dara are available in the Gene Expression Omnibus (GEO) under reference GSE185425. 


\section{References}

Adu MO, Asare PA, Yawson DO, et al. 2017. Quantifying variations in rhizosheath and root system phenotypes of landraces and improved varieties of juvenile maize. Rhizosphere 3, 2939.

Anders S, Huber W. 2010. Differential expression analysis for sequence count data. Genome Biology 11, R106.

Andrews S. 2010. FastQC: a quality control tool for high throughput sequence data. Available online at: https://www.bioinformatics.babraham.ac.uk/projects/fastqc/

Badri D V., Quintana N, El Kassis EG, et al. 2009. An ABC Transporter Mutation Alters Root Exudation of Phytochemicals That Provoke an Overhaul of Natural Soil Microbiota. Plant Physiology 151, 2006-2017.

Baetz U, Martinoia E. 2014. Root exudates: The hidden part of plant defense. Trends in Plant Science 19, 90-98.

Basirat M, Mousavi SM, Abbaszadeh S, et al. 2019. The rhizosheath : a potential root trait helping plants to tolerate drought stress. Plant and Soil 445, 565-575.

Brown LK, George TS, Neugebauer K, et al. 2017. The rhizosheath - a potential trait for future agricultural sustainability occurs in orders throughout the angiosperms. Plant and Soil $418,115-128$.

Burgarella C, Cubry P, Kane NA, et al. 2018. A western Sahara centre of domestication inferred from pearl millet genomes. Nature Ecology \& evolution 2, 1377-1380.

Canarini A, Kaiser C, Merchant A, et al. 2019. Root Exudation of Primary Metabolites: Mechanisms and Their Roles in Plant Responses to Environmental Stimuli. Frontiers in Plant Science 10, 157.

Carvalhais LC, Dennis PG, Fedoseyenko D, et al. 2011. Root exudation of sugars, amino acids, and organic acids by maize as affected by nitrogen, phosphorus, potassium, and iron deficiency. Journal of Plant Nutrition and Soil Science 174, 3-11.

Caye K, Jumentier B, Lepeule J, et al. 2019. LFMM 2: Fast and accurate inference of gene- 
environment associations in genome-wide studies. Molecular Biology and Evolution 36, 852860.

Chai YN, Schachtman DP. 2021. Root exudates impact plant performance under abiotic stress. Trends in Plant Science, in press.

Chakraborty S, Nguyen B, Wasti SD, et al. 2019. Plant Leucine-Rich Repeat Receptor Kinase (LRR-RK): Structure, Ligand Perception, and Activation Mechanism. Molecules 24, 3081 .

Dakora FD, Phillips DA. 2002. Root exudates as mediators of mineral acquisition in lownutrient environments. Plant and Soil 245, 35-47.

De la Fuente Cantó C, Simonin M, King E, et al. 2020. An extended root phenotype: the rhizosphere, its formation and impacts on plant fitness. Plant Journal 103, 951-964.

De-la-Peña C, Badri DV, Zhentian L, et al.. 2010. Root Secretion of Defense-related Proteins Is Development-dependent and Correlated with Flowering Time. Journal of Biological Chemistry 285, 30654-30665.

Debieu M, Kanfany G, Laplaze L. 2017. Pearl Millet Genome: Lessons from a Tough Crop. Trends in Plant Science 22, 911-913.

Debieu M, Sine B, Passot S, et al. 2018. Response to early drought stress and identification of QTLs controlling biomass production under drought in pearl millet. PloS one, 373233.

Delhaize E, James RA, Ryan PR. 2012. Aluminium tolerance of root hairs underlies genotypic differences in rhizosheath size of wheat (Triticum aestivum) grown on acid soil. New Phytologist 195, 609-619.

Delhaize E, Rathjen TM, Cavanagh CR. 2015. The genetics of rhizosheath size in a multiparent mapping population of wheat. Journal of Experimental Botany 66, 4527-4536.

Fang W, Leger RJS. 2010. Mrt, a Gene Unique to Fungi, Encodes an Oligosaccharide Transporter and Facilitates Rhizosphere Competency in Metarhizium robertsii. Plant Physiology 154, 1549-1557.

Feng H, Zhang N, Du W, et al. 2018. Identification of chemotaxis compounds in root exudates 
and their sensing chemoreceptors in plant-growth-promoting rhizobacteria Bacillus amyloliquefaciens SQR9. Molecular Plant-Microbe Interactions 31, 995-1005.

Fernie AR, Martinoia E. 2009. Malate. Jack of all trades or master of a few? Phytochemistry $70,828-832$.

Frichot E, François O. 2015. LEA: An R package for landscape and ecological association studies. Methods in Ecology and Evolution 6, 925-929.

Galloway AF, Akhtar J, Marcus SE, et al. 2020. Cereal root exudates contain highly structurally complex polysaccharides with soil-binding properties. Plant Journal 103, 16661678 .

Gargallo-Garriga A, Preece C, Sardans J, et al. 2018. Root exudate metabolomes change under drought and show limited capacity for recovery. Scientific Reports 8, 1-15.

George TS, Brown L., Ramsay L, et al. 2014. Understanding the genetic control and physiological traits associated with rhizosheath production by barley (Hordeum vulgare). New Phytologist 203, 195-205.

Gong X, McDonald G. 2017. QTL mapping of root traits in phosphorus-deficient soils reveals important genomic regions for improving NDVI and grain yield in barley. Theoretical and Applied Genetics 130, 1885-1902.

Haichar Z, Marol C, Berge O, et al. 2008. Plant host habitat and root exudates shape soil bacterial community structure. The ISME Journal 2, 1221-1230.

Haichar Z, Santaella C, Heulin T. 2014. Soil Biology \& Biochemistry Root exudates mediated interactions belowground. Soil Biology and Biochemistry 77, 69-80.

Hill JT, Demarest BL, Bisgrove BW, et al. 2013. MMAPPR: Mutation Mapping Analysis Pipeline for Pooled RNA-seq. Genome Research 23, 687-697.

Hinsinger P, Bengough AG, Vetterlein D, et al. 2009. Rhizosphere: Biophysics, biogeochemistry and ecological relevance. Plant and Soil 321, 117-152.

Hoover GJ, Van Cauwenberghe OR, Breitkreuz KE, et al. 2007. Characteristics of an Arabidopsis glyoxylate reductase: general biochemical properties and substrate specificity for 
the recombinant protein, and developmental expression and implications for glyoxylate and succinic semialdehyde metabolism in planta. Botany $85,883-895$.

Igamberdiev AU, Eprintsev AT. 2016. Organic acids: The pools of fixed carbon involved in redox regulation and energy balance in higher plants. Frontiers in Plant Science 7, 1042.

James RA, Weligama C, Verbyla K, et al. 2016. Rhizosheaths on wheat grown in acid soils: phosphorus acquisition efficiency and genetic control. Journal of Experimental Botany 67, 3709-3718.

Jarsch IK, Ott T. 2011. Perspectives on Remorin Proteins, Membrane Rafts, and Their Role During Plant-Microbe Interactions. Molecular Plant-Microbe Interactions 24, 7-12.

Kang HM, Zaitlen NA, Wade CM, et al. 2008. Efficient Control of Population Structure in Model Organism Association Mapping. 1723, 1709-1723.

Kawasaki A, Dennis PG, Forstner C, et al. 2021. Manipulating exudate composition from root apices shapes the microbiome throughout the root system. Plant Physiology 0, 1-17.

Kolb E, Legué V, Auvergne UC, et al. 2017. Physical Root-Soil Interactions. Physical Biology 14, 065004.

Lahuta LB, Pluskota WE, Stelmaszewska J, et al. 2014. Dehydration induces expression of GALACTINOL SYNTHASE and RAFFINOSE SYNTHASE in seedlings of pea ( Pisum sativum L.). Journal of Plant Physiology 171, 1306-1314.

Lehle L, Tanner W. 1973. The Function of myo-Inositol in the Biosynthesis of Raffinose. European Journal of Biochemistry 38, 103-110.

Li H, Durbin R. 2009. Fast and accurate short read alignment with burrows-wheeler transform. Bioinformatics 25, 1754-1760.

Lipka AE, Tian F, Wang Q, et al. 2012. GAPIT : genome association and prediction integrated tool. Bioinformatics 28, 2397-2399.

Liu Y, Chen L, Wu G, et al. 2017. Identification of Root-Secreted Compounds Involved in the Communication Between Cucumber, the Beneficial Bacillus amyloliquefaciens, and the Soil-Borne Pathogen Fusarium oxysporum. Molecular Plant- Microbe Interactions 30, 53-62. 
Liu TY, Ye N, Song T, et al. 2019. Rhizosheath formation and involvement in foxtail millet (Setaria italica) root growth under drought stress. Journal of Integrative Plant Biology 61, 449462.

Love MI, Huber W, Anders S. 2014. Moderated estimation of fold change and dispersion for RNA-seq data with DESeq2. Genome Biology 15, 550.

Lynch JP. 2019. Root phenotypes for improved nutrient capture: an underexploited opportunity for global agriculture. New Phytologist 223, 548-564.

Mariac C, Luong V, Kapran I, et al. 2006. Diversity of wild and cultivated pearl millet accessions (Pennisetum glaucum [L.] R. Br.) in Niger assessed by microsatellite markers. Theoretical and Applied Genetics 114, 49-58.

McKenna A, Hanna M, Banks E, et al. 2010. The Genome Analysis Toolkit: A MapReduce framework for analyzing next-generation DNA sequencing data. Genome Research 20, $1297-$ 1303.

Mora-Macías J, Ojeda-Rivera JO, Gutiérrez-Alanís D, et al. 2017. Malate-dependent Fe accumulation is a critical checkpoint in the root developmental response to low phosphate. Proceedings of the National Academy of Sciences of the United States of America 114, E3563E3572.

Moreno-Espíndola IP, Rivera-Becerril F, de Jesús Ferrara-Guerrero M, et al. 2007. Role of root-hairs and hyphae in adhesion of sand particles. Soil Biology and Biochemistry 39, $2520-2526$.

Mwafulirwa L, Baggs EM, Russell JR, et al. 2016. Barley genotype influences stabilization of rhizodeposition-derived $\mathrm{C}$ and soil organic matter mineralization. Soil Biology and Biochemistry 95, 60-69.

Mwafulirwa L, Baggs EM, Russell J, et al. 2021a. Identification of barley genetic regions influencing plant-microbe interactions and carbon cycling in soil. Plant and Soil. https://doi.org/10.1007/s11104-021-05113-6

Mwafulirwa L, Paterson E, Cairns JE, et al. 2021b. Genotypic variation in maize (Zea mays) influences rates of soil organic matter mineralization and gross nitrification. New Phytologist 
$231,2015-2028$.

Nambiar E. 1976. Uptake of Zn65 from dry soil by plants. Plant and Soil 44, 267-271.

Ndour PMS, Barry CM, Tine D, et al. 2021. Pearl millet genotype impacts microbial diversity and enzymatic activities in relation to root-adhering soil aggregation. Plant and Soil 464, 109-129.

Ndour PMS, Gueye M, Barakat M, et al. 2017. Pearl Millet Genetic Traits Shape Rhizobacterial Diversity and Modulate Rhizosphere Aggregation. Frontiers in Plant Science 8, 1288.

Ndour PMS, Heulin T, Achouak W, et al. 2020. The rhizosheath: from desert plants adaptation to crop breeding. Plant and Soil 456, 1-13.

Nguyen K Le, Grondin A, Courtois B, et al. 2019. Next-Generation Sequencing Accelerates Crop Gene Discovery. Trends in Plant Science 24, 263-274.

Nguyen VL, Palmer L, Roessner U, et al. 2019. Genotypic Variation in the Root and Shoot Metabolite Profiles of Wheat ( Triticum aestivum L .) Indicate Sustained, Preferential Carbon Allocation as a Potential Mechanism in Phosphorus Efficiency. Frontiers in Plant Science 10, $1-14$.

Nóbrega FM, Santos IS, Da Cunha M, et al. 2005. Antimicrobial proteins from cowpea root exudates: inhibitory activity against Fusarium oxysporum and purification of a chitinase-like protein. Plant and Soil 272, 223-232.

Omboki RB, Zheng Y, Chen Z, et al. 2018. Pooled mapping of quantitative trait loci conferring male sterility - conditioned glume split in rice ( Oryza sativa ). Plant Breeding 137, $848-856$.

Pang J, Ryan MH, Siddique KHM, Simpson RJ. 2017. Unwrapping the rhizosheath. Plant and Soil 418, 129-139.

Passot S, Gnacko F, Moukouanga D, et al. 2016. Characterization of Pearl Millet Root Architecture and Anatomy Reveals Three Types of Lateral Roots. Frontiers in Plant Science 7, $1-11$. 
Phillips JM, Hayman DS. 1970. Improved procedures for clearing roots and staining parasitic and vesicular-arbuscular mycorrhizal fungi for rapid assessment of infection. Transactions of the British Mycological Society 55, 158-161.

Price SR. 1911. The roots of some North African dessert-glasses. New Phytologist 10, 328340 .

R Core Team. 2018. R: a language and environment for statistical computing. Vienna, Austria: R Foundation for statistical Computing. https://www.R-project.org/.

Rabbi SMF, Tighe MK, Flavel RJ, et al. 2018. Plant roots redesign the rhizosphere to alter the three- dimensional physical architecture and water dynamics. New Phytologist 219, 542550.

Robinson MD, Mccarthy DJ, Smyth GK. 2010. edgeR: a Bioconductor package for differential expression analysis of digital gene expression data. Bioinformatics 26, 139-140.

Sasse J, Martinoia E, Northen T. 2018. Feed Your Friends: Do Plant Exudates Shape the Root Microbiome? Trends in Plant Science 23, 25-41.

Schroth MN, Snyder WC. 1962. Exudation patterns from bean seeds and hypocotyls and their effects on Fusarium solanii f. phaseoli (Abstr.). Phytopathology 52, 751.

Siddappa S, Marathe GK. 2020a. Plant Physiology and Biochemistry What we know about plant arginases? Plant Physiology and Biochemistry 156, 600-610.

Siddappa S, Marathe GK. 2020b. What we know about plant arginases? Plant Physiology and Biochemistry 156, 600-610.

Sung J, Lee S, Lee Y, et al. 2015. Plant Science Metabolomic profiling from leaves and roots of tomato ( Solanum lycopersicum L .) plants grown under nitrogen, phosphorus or potassiumdeficient condition. Plant Science 241, 55-64.

Tesfaye M, Denton MD, Samac DA, Vance CP. 2005. Transgenic alfalfa secretes a fungal endochitinase protein to the rhizosphere. Plant and Soil 269, 233-243.

Trouvelot A, Kough JL, Gianinazzi-Pearson V. 1986. Mesure du taux de mycorhization VA d'un système radiculaire. Recherche de méthode d'estimation ayant une signification 
fonctionnelle. In: Gianinazzi S, Gianinazzi-Pearson V, eds. The Mycorrhizae, Physiology and Genetics. Paris: INRA Presse, 217-221.

Turner T., James EK, Poole P. 2013. The Plant Microbiome. Genome Biology 14, 209.

Varshney RK, Shi C, Thudi M, et al. 2017. Pearl millet genome sequence provides a resource to improve agronomic traits in arid environments. Nature Biotechnology 35, 969-976.

Wissuwa M, Mazzola M, Picard C. 2009. Novel approaches in plant breeding for rhizosphere-related traits. Plant and Soil 321, 409-430.

Yang J, Kloepper JW, Ryu CM. 2009. Rhizosphere bacteria help plants tolerate abiotic stress. Trends in Plant Science 14, 1-4.

York LM, Carminati A, Mooney SJ, et al. 2016. The holistic rhizosphere: integrating zones, processes, and semantics in the soil influenced by roots. Journal of Experimental Botany 67, 3629-3643.

Zhang H, Wang X, Pan Q, et al. 2019. QTG-Seq Accelerates QTL Fine Mapping through QTL Partitioning and Whole-Genome Sequencing of Bulked Segregant Samples. Molecular Plant 12, 426-437.

Zhang Y, Xu F, Ding Y, et al. 2021. Abscisic acid mediates barley rhizosheath formation under mild soil drying by promoting root hair growth and auxin response. Plant, Cell \& Environment 44, 1935-1945. 


\section{Tables}

Table 1. Correlation matrix of root soil aggregation and root parameters.

\begin{tabular}{|c|c|c|c|c|c|c|c|c|c|c|c|c|}
\hline \multirow[b]{2}{*}{ Trait } & \multicolumn{8}{|c|}{ Root architecture } & \multicolumn{2}{|c|}{ Root hairs } & \multicolumn{2}{|c|}{ AM symbiosis } \\
\hline & $\begin{array}{c}\text { RAS/R } \\
\mathbf{T}\end{array}$ & $\mathbf{L}$ & RSA & AvgDiam & LFR & LTR & SAFR & SATR & AvgLRH & AgDRH & $\mathrm{F} \%$ & $\mathbf{I} \%$ \\
\hline RAS/RT & 1 & 0,017 & $-0,081$ & $-0,239$ & 0,053 & $-0,112$ & 0,037 & $-0,110$ & 0,278 & 0,069 & 0,283 & $-0,133$ \\
\hline$p$-value & $\mathbf{0}$ & 0,863 & 0,397 & 0,012 & 0,579 & 0,242 & 0,697 & 0,250 & 0,005 & 0,488 & 0,463 & 0,744 \\
\hline
\end{tabular}

Value from 2 independent experiments on contrasted pearl millet lines using Spearman's correlation test. Ratio (RAS/RT) between the mass of root-adhering soil (RAS) and root tissue biomass (RT), Total root length (L), Root Surface Area (RSA), Average Root Diameter (AvgDiam), Total Length of Fine Roots (LFR), Total Length of Thick Roots (LTR), Surface Area of Fine Roots (SAFR), Surface Area of Thick Roots (SATR), Average Length of Root Hairs (AvgLRH), Average Density of Root Hairs (AgDRH), Frequency of mycorrhization $(\mathrm{F} \%)$, Intensity of mycorrhization ( $\mathrm{I} \%)$.

Table 2. Significant genomic regions identified by Bulk Segregant Analysis (BSA) for rootadhering soil aggregation (i.e. RAS/RT) at the $95 \%$ confidence interval.

\begin{tabular}{cccccc}
\hline & Chr. & Peak position $(\mathrm{Mbp})^{1}$ & Region range $(\mathrm{Mbp})^{2}$ & Region length (Mbp) & Number sig SNPs \\
\hline RAS5.1 & 5 & 3.33 & $0-11.36$ & 11.33 & 3 \\
$R A S 5.2$ & 5 & 112.88 & $92.95-137.95$ & 45.01 & 307 \\
$R A S 5.3$ & 5 & 156.25 & $148.12-158.68$ & 10.56 & 41 \\
\hline RAS6.1 & 6 & 110.41 & $102.08-118.41$ & 16.33 & 16 \\
RAS6.2 & 6 & 226.25 & $218.25-240.48$ & 22.23 & 13 \\
\hline
\end{tabular}

${ }^{1}$ Position of the most significant SNP in the region range

${ }^{2}$ Limits of the significant region considering the overlapping confidence interval of significant markers in the region 


\section{Figure legends}

Figure 1. Relation between root soil aggregation, root architecture, root hair development and arbuscular mycorrhizal symbioses. A) Pearson correlation between traits using adjusted lsmeans across two experiments conducted in different years. B) Linear regression between root diameter and root soil aggregation. Points represent the mean value of the traits for inbred lines across the two experiments. C) Linear regression between root hair length and root soil aggregation. Points represent the mean value of the traits for inbred lines across the two experiments.

Figure 2. Genome-wide association studies (GWAS) for rhizosheath size in pearl millet. Manhattan plots and QQ plots obtained with three GWAS methods. A) Latent Factor Mixed Model or LFMM, B) Mixed linear model or MLM and C) Efficient Mixed Model Association or EMMA. Each Manhattan plot shows the $-\log 10$ p-value of the statistic (y axes) for each SNP position ( $\mathrm{x}$ axes). The dashed line delimits the threshold for highly significant SNPs (pvalue $<10-4)$.

Figure 3. Genetic dissection of root soil aggregation in pearl millet by Bulk Segregant Analysis (BSA). A) Cross established for Bulk Segregant Analysis (BSA) between two pearl millet inbred lines with contrasted rhizosheath phenotype. B) Boxplot showing the distribution of RAS/RT ratio in line ICML-11139 (N=29), ICML-IS $11084(\mathrm{~N}=27)$ and F2 population $(\mathrm{N}=547)$. C) Comparison between GWAS and BSA results. Top figure represents the Manhattan plot of the GWAS by LFMM ridge method (Caye et al., 2019). The x-axis corresponds to the position of the 381,899 SNPs identified by GBS in a group of 139 inbred lines. The vertical axes correspond to the $-\log 10 p$ value of the statistic. The dashed line delimits the threshold for highly significant SNPs ( $p$ value $<10^{-4}$ ). Bottom figure shows the significant regions associated with root soil aggregation identified by BSA using bulks of contrasted F2 lines from a bi-parental cross. The plot shows the Euclidean Distance statistic profile (y axis) across the seven pearl millet chromosomes (x axis). The dashed line indicates the $95 \%$ confidence interval threshold for the localisation of significant regions. In both plots, the shaded area delimits the extent of the five significant regions identified by BSA and the overlap with significant SNPs identified by GWAS and the correspondence with the BSA peaks found. 


\section{Figure 1}

A

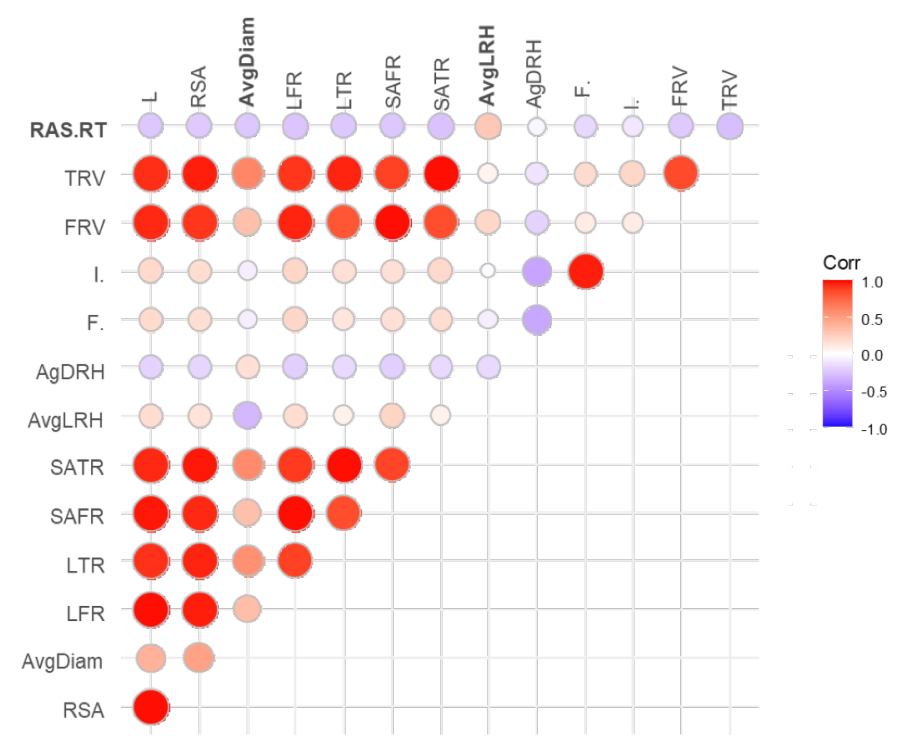

B

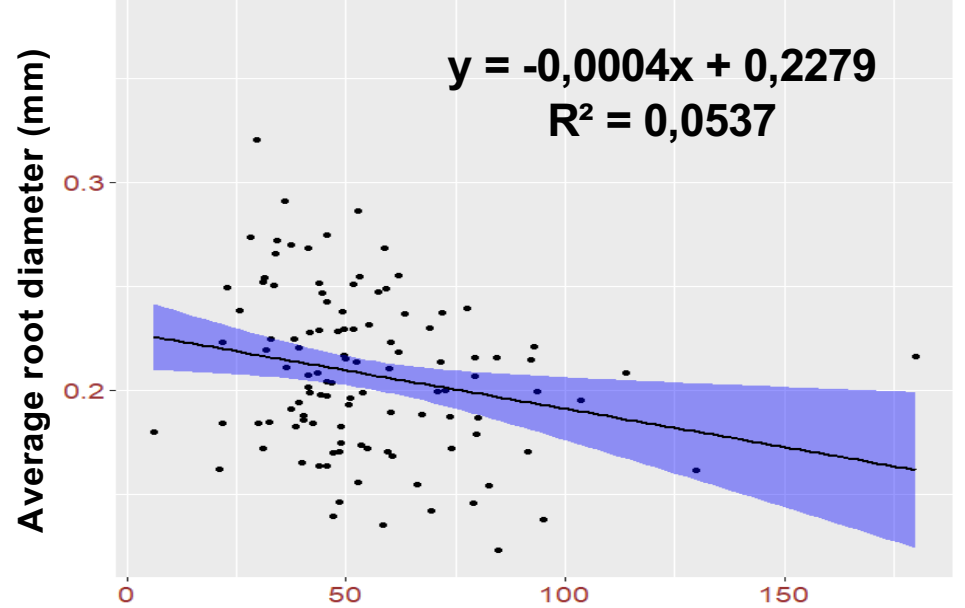

Root soil aggregation (g soil. g-1 $^{-1}$ root)

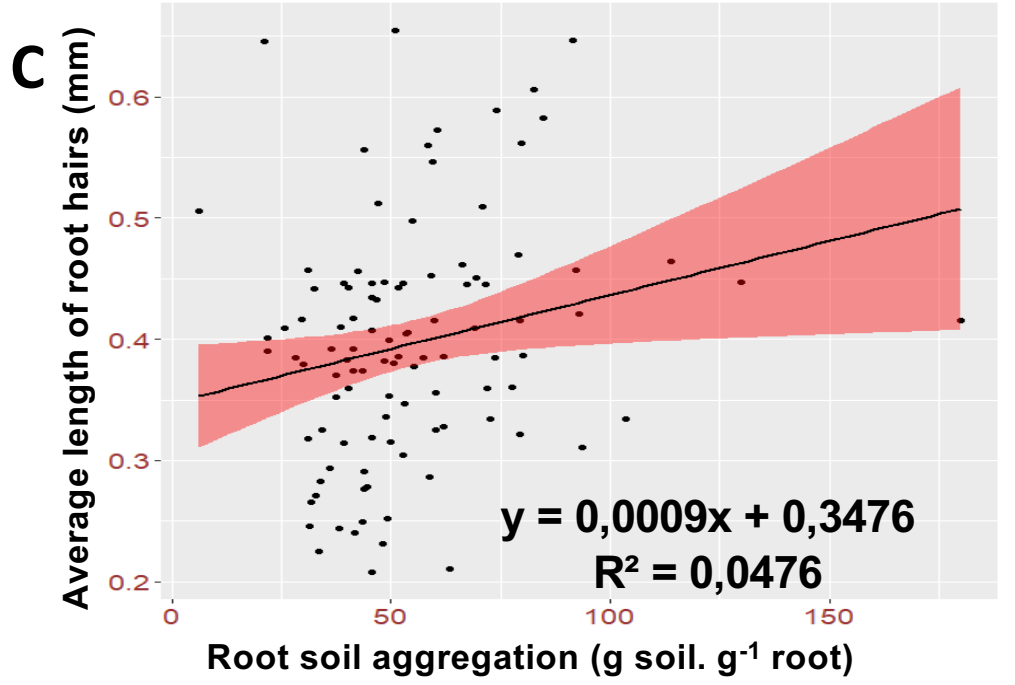


Figure 2 perpetuity. It is made available under aCC-BY-NC-ND 4.0 International license.
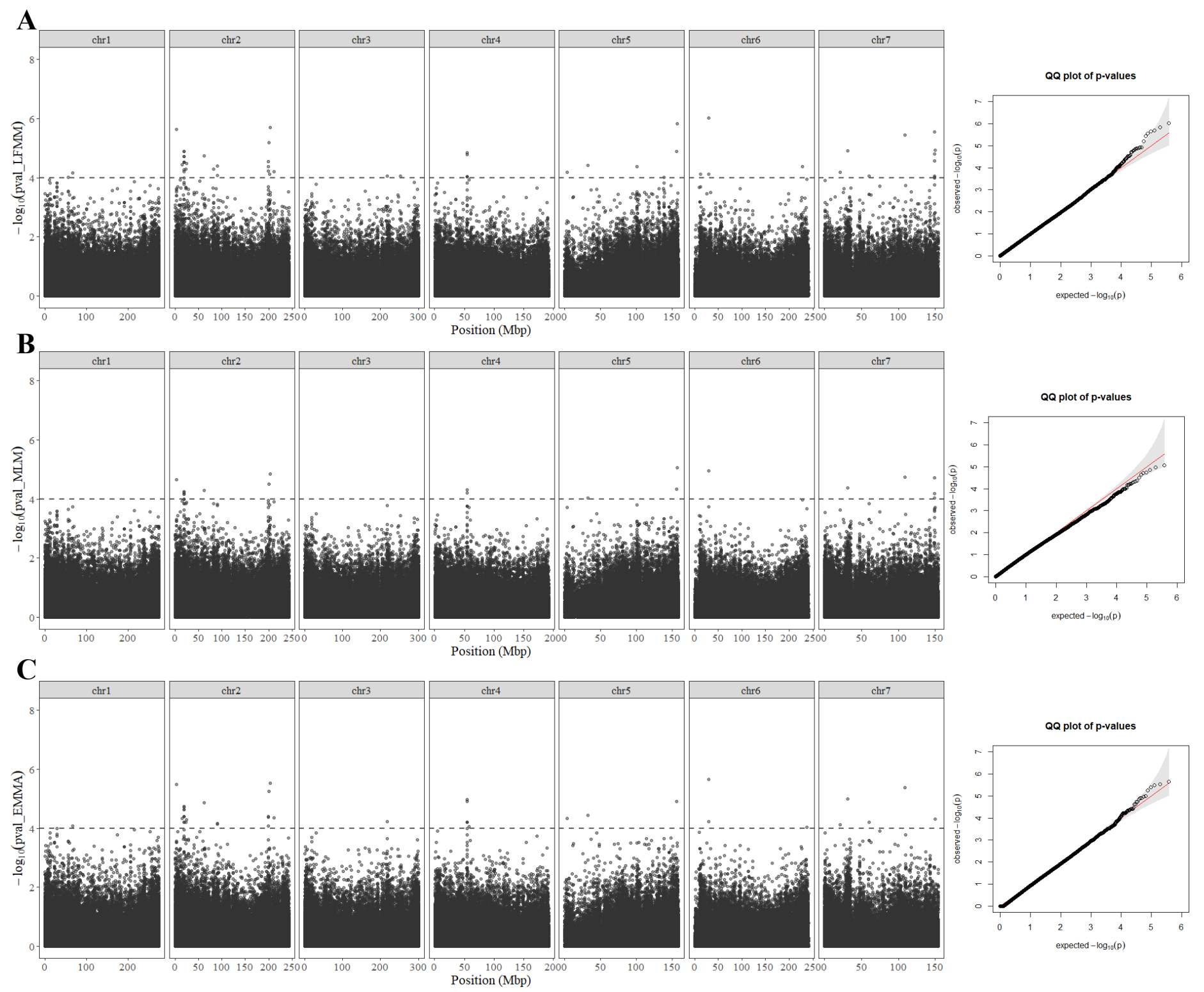

Figure 2. Genome-wide association studies (GWAS) for rhizosheath size in pearl millet. Manhattan plots and QQ plots obtained with three GWAS methods. A) Latent Factor Mixed Model or LFMM, B) Mixed linear model or MLM and C) Efficient Mixed Model Association or EMMA. Each Manhattan plot shows the $-\log 10 p$-value of the statistic (y axis) for each SNP position (x axis). The dashed line delimits the threshold for highly significant SNPs $\left(p\right.$-value $\left.<10^{-4}\right)$. 
bioRxiv preprint doi: https://doi.org/10.1101/2021.11.02.466908; this version posted November $2,2021$. The copyright holder for this preprint (which was not certified by peer review) is the author/funder, who has granted bioRxiv a license to display the preprint in

\section{Figure 3} perpetuity. It is made available under aCC-BY-NC-ND 4.0 International license.

A

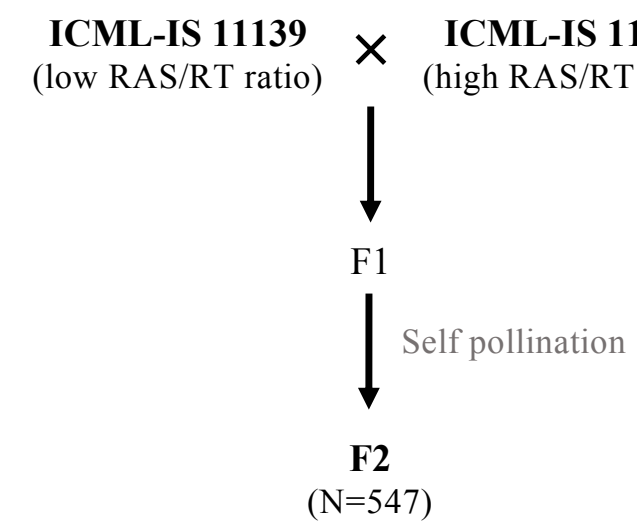

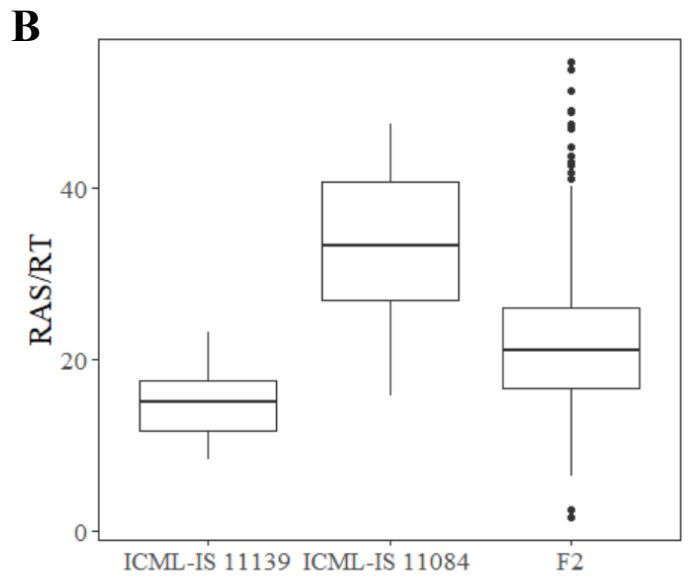
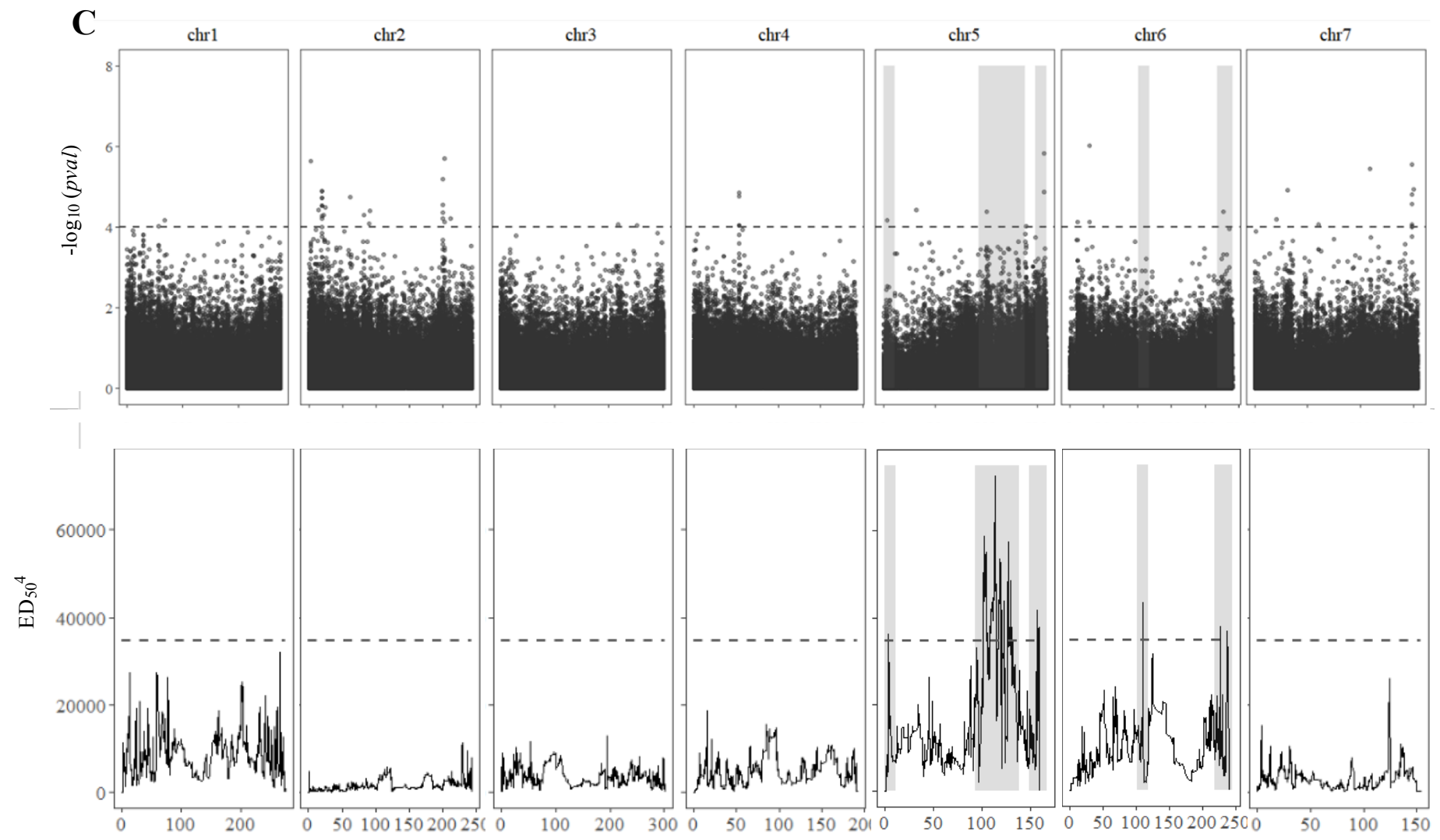

Position (Mbp)

Figure 3. Genetic dissection of root soil aggregation in pearl millet by Bulk Segregant Analysis (BSA). A) Cross established for Bulk Segregant Analysis (BSA) between two pearl millet inbred lines with contrasted rhizosheath phenotype. B) Boxplot showing the distribution of RAS/RT ratio in line ICML-11139 (N=29), ICML-IS $11084(\mathrm{~N}=27)$ and F2 population $(\mathrm{N}=547)$. C) Comparison between GWAS and BSA results. Top figure represents the Manhattan plot of the GWAS by LFMM ridge method (Caye et al., 2019). The horizontal axes corresponds to the position of the 381,899 SNPs identified by GBS in a group of 139 inbred lines. The vertical axes correspond to the $-\log 10$ pvalue of the statistic. The dashed line delimits the threshold for highly significant SNPs $\left(p\right.$ value $\left.<10^{-4}\right)$. Bottom figure shows the significant regions associated with root soil aggregation identified by BSA using bulks of contrasted F2 lines from a bi-parental cross. The plot shows the Euclidean Distance statistic profile (y axes) across the seven pearl millet chromosomes (x axes). The dashed line indicates the $95 \%$ confidence interval threshold for the localisation of significant regions. In both plots, the shaded area delimits the extent of the five significant regions identified by BSA and the overlap with significant SNPs identified by GWAS and the correspondence with the BSA peaks found. 